\title{
Method of Propulsion of a Ferromagnetic Core in the Cardiovascular System Through Magnetic Gradients Generated by an MRI System
}

\author{
Jean-Baptiste Mathieu, Gilles Beaudoin, Member, IEEE, and Sylvain Martel*, Member, IEEE
}

\begin{abstract}
This paper reports the use of a magnetic resonance imaging (MRI) system to propel a ferromagnetic core. The concept was studied for future development of microdevices designed to perform minimally invasive interventions in remote sites accessible through the human cardiovascular system. A mathematical model is described taking into account various parameters such as the size of blood vessels, the velocities and viscous properties of blood, the magnetic properties of the materials, the characteristics of MRI gradient coils, as well as the ratio between the diameter of a spherical core and the diameter of the blood vessels. The concept of magnetic propulsion by MRI is validated experimentally by measuring the flow velocities that magnetized spheres (carbon steel 1010/1020) can withstand inside cylindrical tubes under the different magnetic forces created with a Siemens Magnetom Vision 1.5 T MRI system. The differences between the velocities predicted by the theoretical model and the experiments are approximately $10 \%$. The results indicate that with the technology available today for gradient coils used in clinical MRI systems, it is possible to generate sufficient gradients to propel a ferromagnetic sphere in the larger sections of the arterial system. In other words, the results show that in the larger blood vessels where the diameter of the microdevices could be as large as a couple a millimeters, the few tens of $\mathrm{mT} / \mathrm{m}$ of gradients required for displacement against the relatively high blood flow rate is well within the limits of clinical MRI systems. On the other hand, although propulsion of a ferromagnetic core with diameter of $\sim 600 \mu \mathrm{m}$ may be possible with existing clinical MRI systems, gradient amplitudes of several $\mathrm{T} / \mathrm{m}$ would be required to propel a much smaller ferromagnetic core in small vessels such as capillaries and additional gradient coils would be required to upgrade existing MRI systems for operations at such a scale.
\end{abstract}

Index Terms-Blood vessels, ferromagnetic, magnetic gradients, magnetic resonance imaging system, microdevice, propulsion.

\section{INTRODUCTION}

$\mathbf{M}$ INIMALLY invasive surgery (MIS) can be regarded as a revolution in surgical practices, providing much shorter recovery periods for patients. Although endoscopy and catheterism are currently the most widely used MIS methods,

\footnotetext{
Manuscript received November 11, 2004; revised April 10, 2005. This work was supported in part by a strategic grant from the Natural Sciences and Engineering Research Council of Canada (NSERC), in part by the Canada Research Chair (CRC) in Micro/Nanosystems, Development, Construction, and Validation, Validation Reserche Québec (VRQ), and in part by the Canadian Foundation for Innovation (CFI). Asterisk indicates corresponding author.

J.-B. Mathieu is with Ecole Polytechnique de Montréal (EPM), Montréal, QC H3C 3A7, Canada.

G. Beaudoin is with the University of Montréal, Montréal, QC H3C 3J7, Canada

*S. Martel is with Ecole Polytechnique de Montréal (EPM), Montréal, QC H3C 3A7, Canada (e-mail: sylvain.martel@ polymtl.ca, www.nano.polymtl.ca). Digital Object Identifier 10.1109/TBME.2005.862570
}

many sites in the human body are still inaccessible or at high risk when such modern tools are used. The camera pill $(11 \mathrm{~mm} \times 26$ $\mathrm{mm}$ ) [1] used for the investigation of the gastrointestinal tract and carried by the natural movement of the digestive system is a first good example of the applications of untethered devices in the human body. We can envision that the next step in the evolution of MIS-based applications may come from a new generation of miniature remote wireless controlled devices where maximum dimensions of a few of hundreds of micrometers could be developed in the shorter terms. In many cases, an untethered implementation is suitable in order to reduce the risks of encumbrance of the blood vessels and tissue damages caused by the friction of a tethered wire, especially for complex pathways. Although initially these microdevices would not be capable of performing tasks at a level of complexity equivalent to the catheter, they could be useful for simpler tasks performed in remote sites, which are presently inaccessible to such existing tools. These tasks could include but are not limited to highly localized drug delivery for chemotherapy, thermal treatment of tumors at selected sites, on-site delivery of magnetic resonance imaging (MRI) contrast agents, and carriers for biosensing applications.

When microdevices are propelled in the body fluids, especially in the blood circulatory system, a very large number of remote locations in the human body become accessible. However, since the diameters of the blood vessels in the human body may vary from approximately $25 \mathrm{~mm}$ (Aorta) down to $0.006-0.010$ $\mathrm{mm}$ (capillaries) with diameters of $1.0-4.0 \mathrm{~mm}$ in large arteries, it is obvious that propelling such wireless microdevices in the human cardiovascular system with existing technologies represents a great technical challenge.

Because the method of propulsion should allow such a microdevice to navigate through the cardiovascular system, the use of the normal blood flow itself must be considered only as a complementary means of propulsion when the travel path is in the direction of the blood flow. As such, it is essential to develop a mean of propulsion that could also steer such a microdevice independently from the direction of the blood flow. Several means of propulsion embedded onto such a microdevice have been proposed [2] and include the use of propellers, electromagnetic and jet pumps, membrane propulsion, and active mechanisms to crawl along the surface of the blood vessels. For instance, a crawling mechanism is presented in a recent study that proposes a propulsion system with six embedded actuating legs made of Nafion [3]. However, because of space constraints when operating in the cardiovascular system and the amount of torque required for propulsion against the blood flow, embedding a propulsion mechanism 
is not only a major task, but if feasible, it would restrict significantly the amount of volume available within such microdevices to embed MIS-based functionality.

In order to achieve further miniaturization, the proposed propulsion technique consists of applying magnetic gradients generated by an MRI system to exert a displacement force on a ferromagnetic core embedded onto a miniature untethered device [4], [5]. Since MRI systems generate magnetic gradients and also provide the imaging modality, interfaces, and control within an infrastructure already accessible in clinics and hospitals, the technique is referred here to as magnetic resonance propulsion (MRP) [6]. Other aspects of such MRP systems that are tightly linked to the propulsion have been identified [6] and include primarily the tracking method through MRI-based image distortions generated by the ferromagnetic core [7] and the real-time navigational control of the device within the constraints of a clinical MRI system. Here, only the method of propulsion is addressed in more details.

The use of magnetic gradients to move objects is not recent. One of the first projects, named video tumor fighter (VTF) initiated by the University of Virginia at the end of the eighties [8]-[14] was aimed at moving a ferromagnetic cylinder (thermoseed) through brain tissue so that the cylinder could reach a brain tumor. Once the tumor was penetrated, eddy currents heated the thermoseed. Propulsion of the thermoseed was achieved magnetically by induction coils. The required magnetic field gradients were as high as $5-7 \mathrm{~T} / \mathrm{m}$. Although not used in its original form, the technology is currently applied to deflect ferromagnetic tipped catheters [15].

At Tohoku University, a magnetic propulsion system for small devices in the human body was developed. The system is composed of a screw-shaped cylinder containing a magnet (1-2 $\mathrm{mm}$ in diameter and $8-15 \mathrm{~mm}$ in length). By applying a rotating magnetic field, the device rotates around its main axis and digs its way through tissues. The velocity of the device is a function of the magnetic moment, the number of revolutions of the magnetic field/s and the pitch of the screw [16]-[24].

Following the above examples and our primary studies on using magnetic gradients from an MRI to propel a microdevice in the blood vessels, a team at ETH Zurich recently proposed to use Maxwell coils to generate magnetic field gradients in order to produce propulsion forces for microrobots in body fluids [25].

Although the methods of propulsion using magnetic gradients are not new, the use of a MRI to propel such microdevices is new. Although constrained by the characteristics of clinical MRI systems, this approach offers significant advantages at the system level when compared to other methods. As a matter of fact, MRI system are already implanted in hospitals and provide imaging, tracking, computation, analysis, human interface, gradient coils, cooling systems, real-time navigational control, and several more aspects are tightly linked to the method of propulsion in order to realize a complete and effective system [6].

\section{THEORETICAL MODEL}

As a first approximation, a homogeneous spherical microdevice or core is considered in this paper. The preponderant forces acting on this microdevice are the magnetic force provided by the MRI system and the drag force applied by the blood flow. The weight of the microdevice and its buoyancy can be neglected in small blood vessels because such parameters are smaller than the drag force by several orders of magnitude. This is not applicable for larger devices since the effects of buoyancy and weight become comparable to that of the drag force. The drag force $D$ encompassed by a sphere in an infinite extent of fluid is expressed as

$$
D=\frac{1}{2} \rho_{f} u^{2} A C_{D}
$$

The drag force is a function of fluid density $\rho_{f}$, the relative velocity denoted $u$ between the immersed body and the fluid, the frontal area $A$ of the immersed body, and the drag coefficient $C_{D}$. The drag coefficient $C_{D}$ is a function of the Reynolds number Re [26].

For a spherical body, it is expressed as

$$
\begin{aligned}
C_{D \text { sphere }} & \approx \frac{24}{\operatorname{Re}}+\frac{6}{1+\sqrt{\operatorname{Re}}}+0.4 \\
0 & \leq \operatorname{Re} \leq 2 \times 10^{5}
\end{aligned}
$$

with

$$
\operatorname{Re}=\frac{\rho_{f} u d}{\mu} .
$$

In (3), $d$ is the diameter of the spherical ferromagnetic core and $\mu$ is the viscosity of the fluid.

According to Stokes law of resistance, the drag force in a low Reynolds number $(\operatorname{Re}<1)$ flow is no longer a function of the fluid's density but is linearly proportional to the velocity $u$, viscosity $\mu$ and sphere diameter $d$ [27]

$$
D_{\text {Stokes }}=3 \pi \mu d u ; \quad 0 \leq \operatorname{Re} \leq 1 .
$$

These conditions prevail in the smallest blood vessels (capillaries to small arterioles or venules). In the scope of this paper's experimental conditions, the Reynolds number is comprised between 1000 and 3000. Hence, (1) and (2) will be used instead of Stokes law of resistance.

For endovascular applications, the effect of the blood vessels walls on the drag force has to be taken into account. Although for some blood vessels such as arteries, the diameter of the blood vessels may vary due to cycles of heart contraction, the initial model is simplified by considering the blood vessels as rigid cylindrical tubes.

The drag force acting on a rigid sphere inside a cylindrical tube is a function of $\lambda$ which is the ratio between the sphere's diameter $d$ and the tube's diameter $d_{t}$ as described in [28]-[32]. Many wall effect correlations exist covering many Reynolds numbers ranges. In [30], correlations working for Reynolds numbers from 0.01 to 10000 are proposed by Kehlenbeck and Di Felice. These correlations are very useful to define design rules in the cardiovascular system where $0.001<\operatorname{Re}<10000$. Nevertheless, Munroe proposed a simple empirical correlation to calculate the terminal velocity $u_{t}$ of a sphere with Reynolds number between 1000 and 3000 in a tube and $\lambda<0.6$ in [32]. Since these Re and $\lambda$ conditions are the ones existing in the experimental setup described in this paper, Munroe's simpler 
correlation was used instead of Kehlenbeck and Di Felice's to compare with our data. Munroe's study expressed the wall effect as a ratio between the velocity $u_{t}$ of the sphere in a cylindrical tube and its velocity $u_{t \infty}$ in an infinite extent of fluid. As showed in (5)

$$
\frac{u_{t}}{u_{t \infty}}=1-\lambda^{1.5}
$$

Even though this correlation was developed for a sphere subjected to its own weight, the effect of other forces such as the magnetic force or the buoyancy can be regarded as a change in the density of the sphere.

Once $u_{t \infty}$ and $\mathrm{Re}_{t \infty}$ are known, $C_{D \text { sphere }}$ and $D_{t}$ are computed. The magnetic force required to overcome the drag force acting on a spherical microdevice in a blood vessel can be estimated by applying Newton's third law and taking into account the weight of the microdevice, the buoyancy from the fluid and the drag force at equilibrium

$$
\begin{aligned}
\vec{D}_{t} & =\vec{F}_{m}+\vec{W}+\vec{b} \\
\left|\vec{D}_{t}\right| & =\frac{1}{2} \rho \cdot u_{t \infty}^{2} \cdot A \cdot C_{D_{\text {sphere } \infty}} .
\end{aligned}
$$

In our experimental setup, the Reynolds number and diameter ratio are comprised in the validity range of (5). Hence, this simple calculation method provides an estimation of the magnetic force required for the displacement of the sphere under our experimental conditions.

It is clear that the magnetic force has to be stronger than the drag force to produce displacement against the blood flow. Due to the pulsed nature of the blood flow, the magnetic particle will tend to oscillate while being controlled in the blood vessels. Hence, the control software that is being developed in our laboratory is synchronized with electrocardiogram measurements in order to compute its feedback command. Its refresh rate has to be fast enough to allow a stable control. In clearer terms, it must compute a new command several times/s in order to compensate heartbeat which frequency is around $1 \mathrm{~Hz}$. It is of interest to note that blood flow oscillations are damped by vessels walls and become smaller and smaller as the blood travels further from the heart.

In order to achieve a strong magnetic force, the parameters to be maximized are the magnetization of the microdevice and the magnetic field gradient generated by the gradient coils of the MRI system. The magnetic force is expressed as

$$
\vec{F}_{\text {magnetic }}=R V_{\text {ferro }}(\vec{M} \cdot \nabla) \vec{B} \text {. }
$$

In (7), $F_{\text {magnetic }}$ is the magnetic force (N), $M$ is the magnetization of the material $(\mathrm{A} / \mathrm{m}), V_{\text {ferro }}$ is the volume of the ferromagnetic body $\left(\mathrm{m}^{3}\right), B$ is the magnetic field $(\mathrm{T})$, and $(M \nabla) B$ is the directional derivative of the magnetic field. The term $R$ is the duty cycle representing the time that the magnetic gradients are applied within each cycle as described later in more details.

Clinical MRI systems currently provide tens of $\mathrm{mT} / \mathrm{m}$ of gradients in any direction for imaging purposes. Such gradients can also be used to produce a magnetic force vector in any directions through the magnetic coils. To produce a strong magnetic force, magnetic materials having strong magnetization are required. In this context, ferromagnetic materials are suitable because they have the strongest magnetization with a saturation value represented as $M_{\text {sat }}$ which is higher than other magnetic materials. The amplitude of $M_{\text {sat }}$ is the most important magnetic characteristics for propulsion since typical $1.5 \mathrm{~T}$ magnetic fields found in clinical MRI systems are strong enough to saturate a ferromagnetic core. Permendur with the highest saturation magnetization $\left(M_{\mathrm{sat}}=1.950 \times 10^{6} \mathrm{~A} / \mathrm{m}\right)$ is of particular interest in this application since it could provide the highest magnetic force/unit of volume.

\section{EXPERIMENTS}

Experiments were conducted in order to measure the influence of magnetic field gradients from an MRI system on a ferromagnetic sphere. The experimental setup consisted of a cylindrical rigid polymethylmethacylate (PMMA) tube containing the ferromagnetic sphere acting against a flow of water circulating though the tube. For each experiment, the tube was oriented along the $x, y$ and $z$ directions within the bore of a $1.5 \mathrm{~T}$ Siemens Magnetom Vision MRI scanner capable of providing maximum gradients of $25 \mathrm{mT} / \mathrm{m}$. This paper focuses on the results obtained along the $y$ direction or vertical axis since these data are the most relevant in terms of the effect of weight and buoyancy. Data obtained along the horizontal directions can be found in our previous studies [4], [33].

During propulsion, the excess of heat in the coils is also a constraint. The cooling system for the gradient coils in a standard MRI system is designed for imaging purpose and as such, it cannot evacuate the excess of heat generated by the coils when used continuously at maximum amplitude. When used to propel a ferromagnetic core, a larger duty cycle is required compared to imaging and thus, the temperature of the coils is reduced during the experiments by applying lower gradient amplitudes in order to increase the duty cycle.

The ferromagnetic spheres used for the experiments were made of 1010/1020 carbon steel with a diameter of $3.14 \mathrm{~mm}$. The saturation magnetization of this alloy is $M_{\text {sat }}=1.376 \times$ $10^{6} \mathrm{~A} / \mathrm{m}$ with a saturating magnetizing induction of $B_{\mathrm{sat}}=$ 0.734 T. These values were measured with a Princeton Applied Research Corporation 155 vibrating sample magnetometer [34] and an F.W. Bell 610 Hall Effect Gaussmeter [35]. These spheres were saturated when placed inside the $1.5 \mathrm{~T}$ MRI system $\left(B_{0}=1.5 \mathrm{~T}>B_{\text {sat }}=0.734 \mathrm{~T}\right)$. A pump (March MDXT-3) [36] and a variable area flowmeter [37] equipped with a control valve were placed outside the room where the MRI system was installed. Inside the MRI bore, the flexible PVC tubing was connected to a straight cylindrical PMMA tube with a diameter of $6.65 \mathrm{~mm}$. The PMMA tube was placed vertically inside the MRI bore ( $y$ direction). The tube was supported by three PMMA plaques that could be oriented independently to adjust the tube along the vertical axis. For each experiment, a 1010/1020 carbon steel sphere was placed inside the PMMA tube with the water flowing upward. One filter was placed at each extremity of the tube to maintain the sphere within its boundaries. A second valve was placed at the outlet of the PMMA tube and was used to adjust the water flow. This valve was completely made of plastic since it was placed inside the MRI bore. Fig. 1 shows photographs of the experimental setup. 


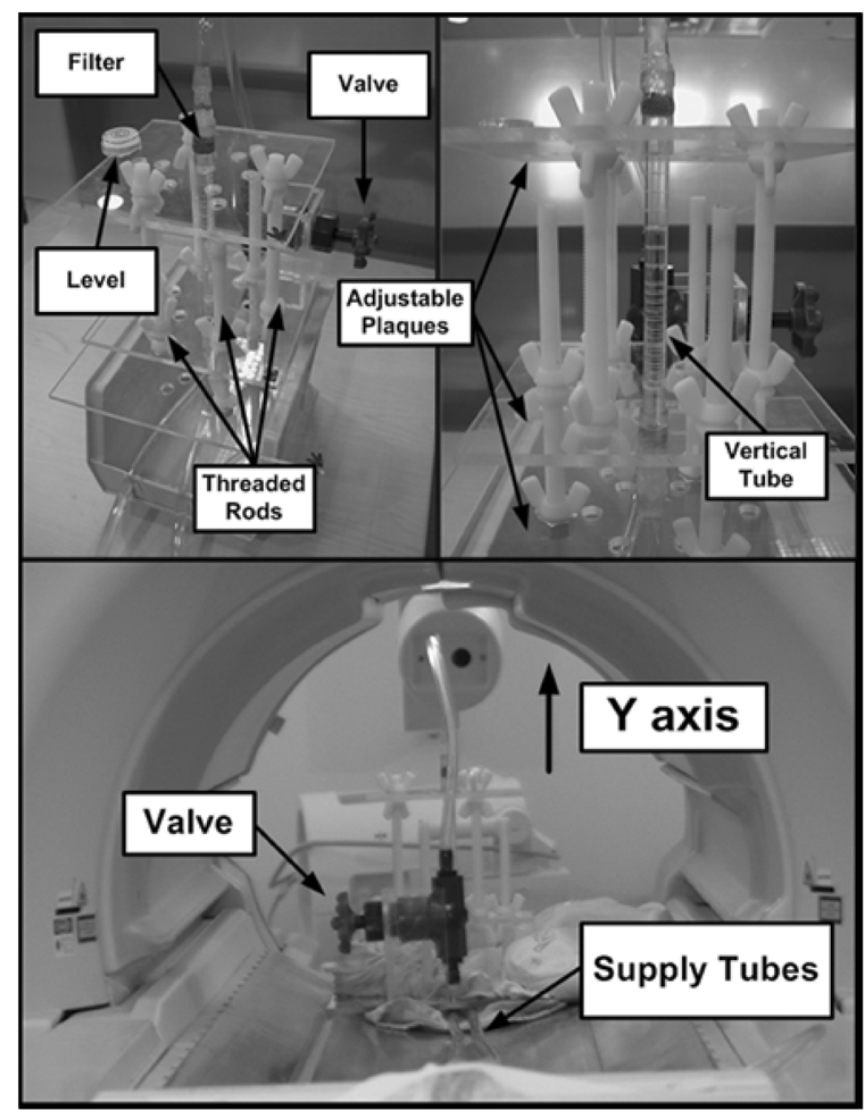

Fig. 1. Photographs of the experimentation setup showing the vertical tube where the ferromagnetic sphere was placed and the orientation of the setup inside the MRI bore.

During the experiments, the ferromagnetic sphere was under the influence of the drag force $D$, propulsion magnetic force $F_{\text {MagPropy }}$, the buoyancy $b$ and of its own weight $W$. The following gradients were applied: $18 \mathrm{mT} / \mathrm{m}, 16 \mathrm{mT} / \mathrm{m}, 14 \mathrm{mT} / \mathrm{m}$, $12 \mathrm{mT} / \mathrm{m}, 9 \mathrm{mT} / \mathrm{m}, 8 \mathrm{mT} / \mathrm{m}, 7 \mathrm{mT} / \mathrm{m},-7 \mathrm{mT} / \mathrm{m},-8 \mathrm{mT} / \mathrm{m}$, $-9 \mathrm{mT} / \mathrm{m},-12 \mathrm{mT} / \mathrm{m},-14 \mathrm{mT} / \mathrm{m},-16 \mathrm{mT} / \mathrm{m}$, and $-18 \mathrm{mT} / \mathrm{m}$. A positive gradient generates an upward magnetic force while a negative gradient generates a downward magnetic force. For each gradient value, a duty cycle $R$ of $83.3 \%$ was used with a $12-\mathrm{ms}$ period (i.e., $10 \mathrm{~ms}$ of continuous applied gradient within each period of $12 \mathrm{~ms}$ ) and other experiments were performed with a continuous $5 \mathrm{~ms}$ of gradients $(41.667 \%$ duty cycle). In the initial phase, the position of the ferromagnetic sphere was stabilized and the water flow was measured prior to the application of a gradient. Once the gradient was applied, the position of the ferromagnetic sphere was stabilized again and the new water flow rate was measured. For each gradient-duty cycle combination, the equilibrium water flows were measured three times and averaged. The mean water velocity $u_{t}(\mathrm{~m} / \mathrm{s})$ was calculated by dividing the equilibrium flow measurement $Q\left(\mathrm{~m}^{3} / \mathrm{s}\right)$ by the tube's cross sectional area $A\left(\mathrm{~m}^{2}\right)$ according to $Q=u_{t} A$.

The mean water velocity without magnetic force was measured as $441.35 \pm 5.51 \mathrm{~mm} / \mathrm{s}$. In order to increase the quality of the data, the measurements were normalized according to the mean water velocity without magnetic force. The experimental

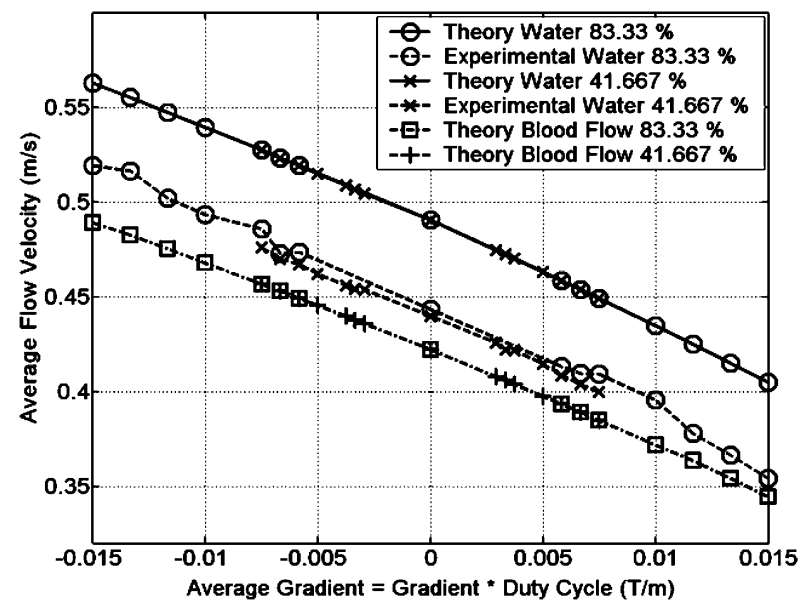

Fig. 2. Experimental average flow velocities versus applied gradients compared with theoretical flow velocities for water and blood. There is a good correlation between the values with a duty cycle of $83.33 \%$ and of $41.667 \%$ for the equivalent gradients.

setup aimed at simulating peripheral arteries and as such, the diameter of the tube and the corresponding flows were chosen to be of the same order of magnitude as the ones in adult's arteries.

\section{RESULTS}

In Fig. 2, the velocity measurements for each duty cycle $R$ and magnetic field gradient combination is compared with the output of the theoretical model described previously for water and for blood. Experimental data are plotted by weighting the gradient amplitudes by the corresponding duty cycle. As shown in Fig. 2, a standard MRI system can generate enough magnetic force to propel a ferromagnetic sphere inside a flow in a duct with properties close to the ones found in real arteries in terms of diameter, fluid density, flow, and Reynolds number. Water was used instead of blood in these experiments. The reason for this choice was the necessity to use a transparent fluid for monitoring by visual means. Blood's density and viscosity being, respectively, 1.05 and 3.5 times higher than water, the theoretical prediction of the use of blood instead of water in our experiments taking these properties in account is also plotted in Fig. 2.

This vertical experimental setup allows the measurements to be freed from the influence of friction between the sphere and tube walls. It simulates an endovascular navigation case where the gradient coils would be strong enough to levitate the sphere $(\nabla \vec{B} \approx 60 \mathrm{mT} / \mathrm{m})$.

For the experiments, two different duty cycles were used to confirm the relation between magnetic force and the term $R$ in (7). The experimental results indicate that to make the ferromagnetic spheres withstand the same water flow rates, applying gradients of 18,16 , and $14 \mathrm{mT} / \mathrm{m}$ with $R=41.667 \%$ gave the same results as using gradients of 9,8 , and $7 \mathrm{mT} / \mathrm{m}$, respectively, with $R=83.33 \%$ (measurements are less than 1 standard deviation away).

The theoretical values plotted in Fig. 2 were calculated from Munroe's correlation [32] by equating the drag force with the vector sum of the sphere's weight $W$, buoyancy $b$, and magnetic force $F_{\text {magnetic }}$, and weighted according to the duty cycle 
$R$. Munroe's correlation relies on measurements of the equilibrium velocity of spheres suspended in a tube and subjected to rising flows of water. The velocity of the flow is greatest in the center of the tube, and least at the walls, where it is retarded by friction. Hence, the smaller a sphere is compared to the diameter of the tube, the more it tends to drift radially in the tube. In Munroe's experiments, the spheres were physically constrained to remain centered in the tube. It was not the case in our experiments, where the sphere was free to drift radially in the tube. The differences between the theoretical calculations and the experimental data are $9.36 \%$ and $10.40 \%$ for $R=83.33 \%$, and $R=41.667 \%$, respectively. These differences are strongly dependent upon experimental conditions. We attribute them to experimental imprecisions due to fluctuations in drag force when the sphere drifts radially in the tube. In the human body, the velocity profile of the blood flow is different in every blood vessel with fluctuations over time. In this paper, a simple flow model such as Munroe's was used to compare with our data from the experimental setup.

The shape of the immersed body is of primary importance. An ellipsoid for instance, would experience a lower drag force than a sphere with the same volume providing the capability to withstand a higher flow rate since its drag coefficient $C_{D}$ would be lower [38]. However, since a magnetized body tends to remain oriented in the direction of the magnetic field, core geometries such as ellipsoids or other nonspherical shapes can be a problem when navigating in tubes or blood vessels with complex geometries, in particular through curvatures with a relatively small radius.

\section{DISCUSSION}

Other existing clinical MRI systems (for an example, see [39]) can generate $66 \mathrm{mT} / \mathrm{m}$ gradients, inducing up to 3.66 times more magnetic force (with $100 \%$ duty cycle) than the MRI system used in our experiments. Using such a system with a ferromagnetic sphere of the same dimensions but made of Permendur could generate up to 5.37 times more magnetic force when a duty cycle of $100 \%$ is used. Theoretical calculations predict that a Permendur sphere would have been able to withstand a $0.71 \mathrm{~m} / \mathrm{s}$ water mean velocity under the same experimental conditions.

The proposed theoretical model was validated by comparison with other experimental studies [28], [32] and with the data obtained from our MRI-based propulsion experiments. This model allows us to evaluate the propulsion force required for ferromagnetic core of different dimensions to be propelled in blood vessels of different diameters.

The magnetic force $\vec{F}_{\text {Mag }}$ from an MRP system must have two vectored components

$$
\begin{aligned}
\vec{F}_{\mathrm{Mag}} & =\vec{F}_{\mathrm{MagLev}}+\vec{F}_{\mathrm{MagProp}} \\
\vec{F}_{\mathrm{MagLev}} & =-(\vec{W}-\vec{b})=-\vec{W}_{a} .
\end{aligned}
$$

In a horizontal blood vessel for instance, a magnetic force $F_{\text {MagLev }}$ is required for levitation to detach the ferromagnetic body from the lower wall of the blood vessel while the magnetic propulsion force $F_{\text {MagProp }}$ (determined by estimating the a)

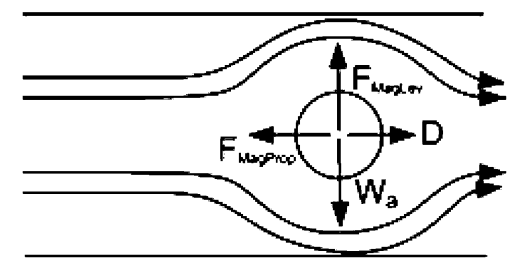

b)
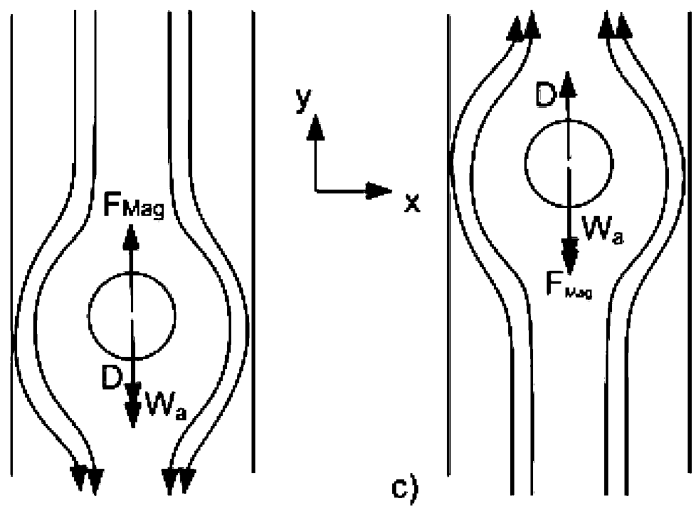

Fig. 3. Effect of apparent weight of a ferromagnetic core on the magnetic force required for displacement. (a) In a horizontal tube, the drag force and the horizontal component of the magnetic force are in opposite direction to each other. A vertical magnetic force is used to levitate the magnetic sphere. (b) In a vertical tube and a downward flow, the magnetic force must be greater than the sum of the weight of the sphere and the drag force for a movement in the upward direction. (c) In a vertical tube and an upward flow, the weight of the magnetic sphere contributes to the propulsion against the flow.

drag force on the ferromagnetic body) allows the ferromagnetic body to move against the blood flow. In the above equations, $W$ is the weight of the ferromagnetic device, $b$ is its buoyancy, and $W_{a}$ the apparent weight. $W_{a}$ can be a disadvantage in the case where the ferromagnetic device has to move upward, requiring an increase of the magnetic force $F_{\text {Mag. }}$. But if the ferromagnetic core has to move downward, $W_{a}$ would contribute to the movement and the required magnetic force $F_{\text {Mag }}$ would be reduced accordingly as shown in Fig. 3.

In the conditions of Fig. 4, the drag force is stronger than the weight for small ratios of sphere/tube diameters. When the diameter ratio is increased, the weight of the ferromagnetic sphere becomes higher than the drag force until the wall effect becomes predominant. There is a region of sphere/tube diameter ratios where the weight of the sphere compared to the drag force is optimized and where higher terminal velocities can be reached.

In Fig. 4, the horizontal line corresponds to the unit force ratio. Between the two intersection points, the weight is stronger than the drag force and sphere moves downward if it is placed at a $1 \mathrm{~m} / \mathrm{s}$ blood flow in a $2.5-\mathrm{cm}$ tube. On the left and right hand side of the intersection points, the sphere is carried away by the blood flow. The optimal diameter ratio $\lambda_{\mathrm{Optim}}$ is the minimum of the $f=D / W$ curve. In this case, it is $\lambda_{\text {Optim }}=0.42$.

In our particular application, the forces acting on the ferromagnetic sphere are the drag force $D$, the weight $W$, the buoyancy $b$, and the magnetic force $F_{\mathrm{Mag}}$. The last three forces are proportional to the volume of the sphere that is to say, to the cube of its radius. Hence, a sphere subjected to any combinations of these forces will show a similar optimal diameter than the one 


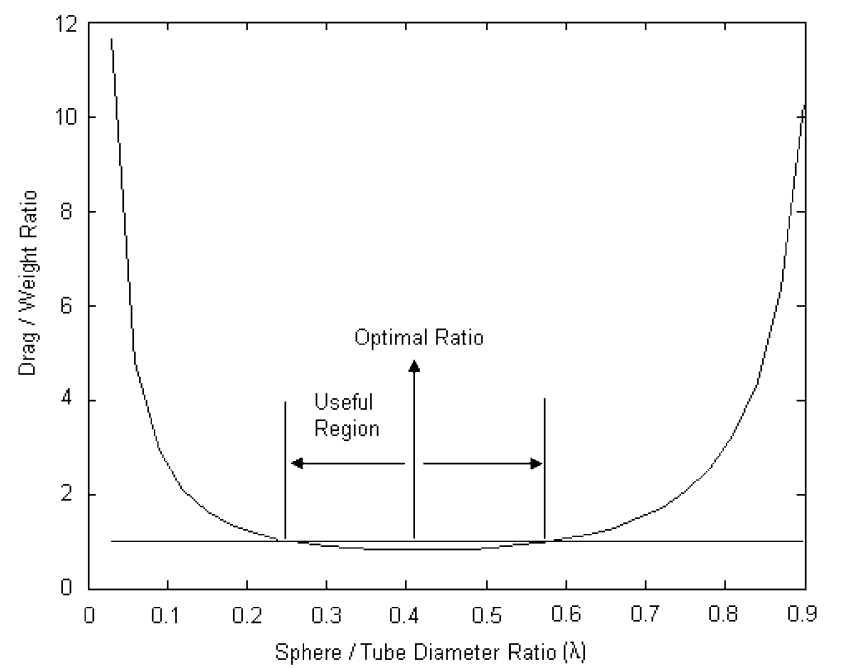

Fig. 4. Drag/weight ratio as a function of the sphere/tube ratio. The diameter of the tube is $2.5 \mathrm{~cm}$, blood is considered as the fluid with flow velocity of 1 $\mathrm{m} / \mathrm{s}$. Optimal sphere/tube ratio is $\lambda=0.42$.

computed for the falling sphere. The optimal sphere/tube diameter ratio is only a function of the cube of the radius. Hence, the $f=\operatorname{drag} / \Sigma$ (volumicforces) and $f=\mathrm{drag} / r^{3}$ both reach their minima with the same sphere/tube ratio. Therefore, the $f=\mathrm{drag} / r^{3}$ function can be used to determine the optimal sphere radius for a given diameter of blood vessel and the corresponding blood flow parameters.

Based on our previous results, Fig. 5 shows a prediction of the magnetic field gradients that would be required to navigate a potential microdevice in the cardiovascular system. To be more realistic, the prediction assumes that $50 \%$ of the volume of the spherical microdevice is made of Permendur $\left(M_{\text {Sat }}=1.9496 \times 10^{6} \mathrm{~A} / \mathrm{m}\right)$ leaving $50 \%$ of the volume for the target application. The microdevice diameters that are used in calculations of Fig. 5, are chosen to optimize $\lambda$ when the diameter of the blood vessel is fixed. In Fig. 5, propulsion gradients correspond to an average value over the gradient period, i.e., a $100 \mathrm{mT} / \mathrm{m}$ gradient with a duty cycle of $50 \%$ is equivalent to a gradient of $50 \mathrm{mT} / \mathrm{m}$ with a duty cycle of $100 \%$.

In Fig. 5, to navigate in the aorta where the diameter of the blood vessel is in the order of $25 \mathrm{~mm}$ with a maximum blood flow velocity of $\sim 100 \mathrm{~cm} / \mathrm{s}$, a spherical microdevice with an overall diameter of $10.5 \mathrm{~mm}$ containing $50 \%$ Permendur would require a minimum of $\sim 70 \mathrm{mT} / \mathrm{m}$ with $100 \%$ duty cycle and adjusted to the change in velocity of the blood flow. An increase of the propulsion force could potentially be obtained by increasing the percentage of Permendur in the core for some applications. In veins and arteries with an inside diameter of the blood vessel of approximately 4-5 mm and a maximum average blood flow (not as pulsatile as the Aorta) of $0.3-5 \mathrm{~cm} / \mathrm{s}$ and $10-40 \mathrm{~cm} / \mathrm{s}$ for the veins and arteries, respectively, a very small gradient amplitude would be required for the veins but problematic in the case where the flow increases to more than approximately $30 \mathrm{~cm} / \mathrm{s}$ in arteries depending on the duty cycle used. In large veins (5-30 $\mathrm{mm}$ inner diameter), the average blood velocity is between 15 and $20 \mathrm{~cm} / \mathrm{s}$ with a maximum blood velocity between 50 and $90 \mathrm{~cm} / \mathrm{s}$. In such a case, the required gra-

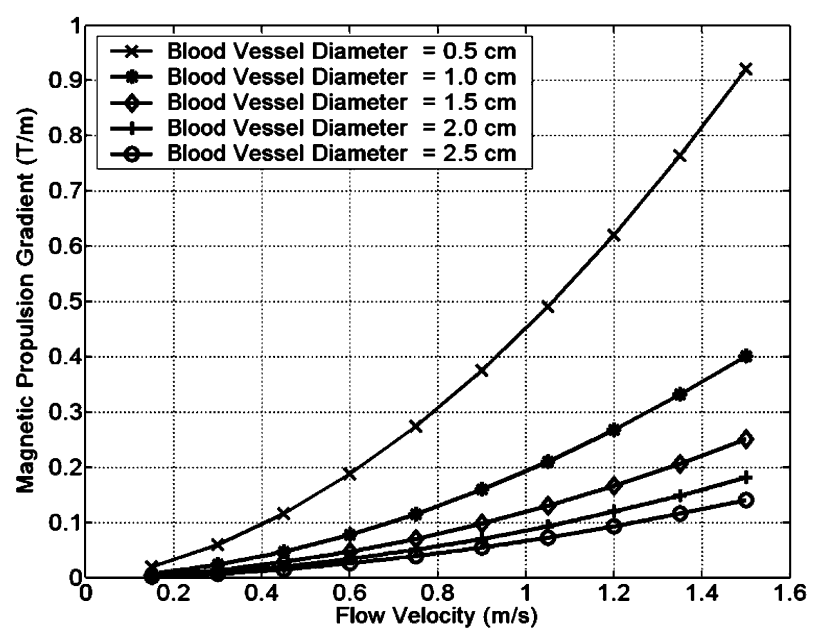

Fig. 5. Optimized magnetic gradient of propulsion in 5 tubes versus the flow velocity.

dient is $\sim 50 \mathrm{mT} / \mathrm{m}$ and 50 to $100 \mathrm{mT} / \mathrm{m}$ for arteries $(2-6 \mathrm{~mm}$ inner diameter) with maximum blood velocities between 20 and $50 \mathrm{~cm} / \mathrm{s}$, depending on the concentration of ferromagnetic material in the core.

It is important to specify that in much smaller diameter blood vessels, the problem of propulsion is quite different. First, blood velocity decreases from more than 1 meter/s in the aorta to less than $1 \mathrm{~mm} / \mathrm{s}$ in the capillaries. Moreover, in small blood vessels, high shear rates and the Fahraeus-Lindqvist effect [40] tend to reduce blood's viscosity and hence the Reynolds number Re increases and the drag coefficient $C_{D}$ decreases accordingly. Furthermore, the gradients required to navigate a ferromagnetic core in small blood vessels such as capillaries for instance, are beyond the gradients that a clinical MRI system can provide without the addition of a gradient coil especially designed to provide such propulsion gradients. As such, the model described in this paper assumes a larger sphere propelled in a corresponding larger blood vessel.

\section{CONCLUSION}

In this paper, we introduce the first attempt to use an MRI system as a mean of propulsion for a ferromagnetic body. The proposed mathematical model conforms relatively well with the results of the experiments and indicates that a clinical MRI scanner could be used to generate enough magnetic force to move a ferromagnetic body in the cardiovascular system. Finally, it was found that an additional gradient coil could be necessary to provide enough magnetic field gradients for the navigation of ferromagnetic cores in smaller blood vessels.

\section{ACKNOWLEDGMENT}

The authors wish to acknowledge the support of K. Kim for his help in reviewing this manuscript and other members that contributed to other aspects of this project not described in this paper. They also acknowledge the support of Hospital Notre-Dame and Centre Hospitalier de l'Université de Montréal (CHUM) that provided the infrastructure necessary to conduct MRI tests. 


\section{REFERENCES}

[1] (2004) Given Imaging. Given Imaging Home Page. [Online]. Available: http://www.givenimaging.com

[2] L. Rubinstein. A practical NanoRobot for treatment of various medical problems. presented at 8th Foresight Conf. Molecular Nanotechn.. [Online]. Available: http://www.foresight.org/Conferences/MNT8/Papers/Rubinstein/

[3] M. Otis, R. Bernier, Y. Pasco, H. Menard, H. Semmaoui, M. Jarry, and R. Fontaine, "Development of an Hexapod BioMicRorobot with Nafion-Pt IPMC microlegs," in Proc. 25th Ann. Int. Conf. IEEE Engineering in Medicine and Biology Society, Cancun, Mexico, 2003, pp. 3423-3426.

[4] J.-B. Mathieu, S. Martel, L. Yahia, G. Soulez, and G. Beaudoin, "MRI systems as a mean of propulsion for a microdevice in blood vessels," in Proc. 25th Ann. Int. Conf. IEEE Engineering in Medicine and Biology Society, Cancun, Mexico, 2003, pp. 3419-3422.

[5] S. Martel, J. Mathieu, L. Yahia, G. Beaudoin, and G. Soulez, "Method and System for Propelling and Controlling Displacement of a Microrobot in a Blood Vessel," US Patent 10/417 475, Apr. 15, 2003.

[6] S. Martel, J.-B. Mathieu, O. Felfoul, H. Macicior, G. Beaudoin, G. Soulez, and L. Yahia, "Adapting MRI systems to propel and guide microdevices in the human blood circulatory system," presented at the 26th Conf. IEEE Engineering in Medicine and Biology Society, San Francisco, CA, Sep. 2004.

[7] O. Felfoul, S. Martel, G. Beaudoin, and J.-B. Mathieu, "Micro-device's susceptibility difference based MRI positioning system, a preliminary investigation," presented at the 26th Conf. IEEE Engineering in Medicine and Biology Society, San Francisco, CA, Sep. 2004.

[8] M. S. Grady, M. A. Howard 3rd, J. A. Molloy, R. C. Ritter, E. G. Quate, and G. T. Gillies, "Nonlinear magnetic stereotaxis: Three-dimensional, in vivo remote magnetic manipulation of a small object in canine brain," Med. Phys., vol. 17, pp. 405-15, May-Jun. 1990.

[9] M. S. Grady, M. A. Howard, R. C. Ritter, E. G. Quate, J. A. Molloy, M. A. Lawson, K. G. Wika, P. A. Ramos, and G. T. Gillies, "Magnetic stereotaxis system for neurosurgical procedures," in Instrum. Aerosp. Ind., Proc. ISA Aerospace Instrumentation Symp.: Proc. 37th Int. Instrumentation Symp., vol. 37, May 5-9, 1991, pp. 665-675.

[10] M. S. Grady, M. A. Howard 3rd, W. C. Broaddus, J. A. Molloy, R. C. Ritter, E. G. Quate, and G. T. Gillies, "Magnetic stereotaxis: A technique to deliver stereotactic hyperthermia," Neurosurgery, vol. 27, pp. 1010-5, Dec. 1990.

[11] M. S. Grady, M. A. Howard 3rd, J. A. Molloy, R. C. Ritter, E. G. Quate, and G. T. Gillies, "Preliminary experimental investigation of in vivo magnetic manipulation: Results and potential application in hyperthermia," Med. Phys., vol. 16, pp. 263-72, Mar.-Apr. 1989.

[12] J. A. Molloy, R. C. Ritter, M. S. Grady, M. A. Howard 3rd, E. G. Quate, and G. T. Gillies, "Experimental determination of the force required for insertion of a thermoseed into deep brain tissues," Ann. Biomed. Eng., vol. 18, pp. 299-313, 1990.

[13] M. A. Howard, M. S. Grady, R. C. Ritter, G. T. Gillies, E. G. Quate, and J. A. Molloy, "Magnetic movement of a brain thermoceptor," Neurosurgery, vol. 24, pp. 444-8, Mar. 1989.

[14] E. G. Quate, K. G. Wika, M. A. Lawson, G. T. Gillies, R. C. Ritter, M. S. Grady, and M. A. I. Howard, "Goniometric motion controller for the superconducting coil in a magnetic stereotaxis system," IEEE Trans. Biomed. Eng., vol. 38, no. 9, pp. 899-905, Sep. 1991.

[15] (2004) Stereotaxis. Stereotaxis—Digital Solutions for Interventional Medicine. [Online]. Available: http://www.stereotaxis.com

[16] T. Honda, K. I. Arai, and K. Ishiyama, "Micro swimming mechanisms propelled by external magnetic fields," in IEEE Trans. Magn. (Proc. 1996 IEEE Int. Magnetics Conf. (INTERMAG'96). Part 2 (of 3), vol. 32, Sep. 1996, pp. 5085-5087.

[17] K. Ishiyama, K. I. Arai, M. Sendoh, and A. Yamazaki, "Spiral-type micro-machine for medical applications," in Proc. 2000 Int. Symp. Micromechatronics and Human Science, Oct. 22-25, 2000, pp. 65-69.

[18] K. Ishiyama, M. Sendoh, and K. I. Arai, "Magnetic micromachines for medical applications," J. Magn. Magn. Mater, vol. 242-245, no. I, pp. 41-46, Apr. 2002.

[19] K. Ishiyama, M. Sendoh, A. Yamazaki, and K. I. Arai, "Swimming micro-machine driven by magnetic torque," Sensors Actuators, A: Phys., vol. 91, no. 1-2, pp. 141-144, Jun. 2001.
[20] K. Ishiyama, M. Sendoh, A. Yamazaki, M. Inoue, and K. I. Arai, "Swimming of magnetic micro-machines under a very wide-range of Reynolds number conditions," in Proc. 8th Joint Magnetism and Magnetic Materials Int. Magnetic Conf. (MMM-Intermag), Jan. 7-11, 2001, pp. 2868-2870.

[21] M. Sendoh, N. Ajiro, K. Ishiyama, M. Inoue, K. I. Arai, T. Hayase, and J. Akedo, "Effect of machine shape on swimming properties of the spiral-type magnetic micro-machine," in 1999 IEEE Int. Magnetics Conf. (IEEE Intermag 1999), May 18-21, 1999, pp. GF-10-GF-10.

[22] M. Sendoh, K. Ishiyama, M. Inoue, and K. I. Arai, "Fabrication of spiral-type magnetic micro-machine running in a gel," in 2000 IEEE Int. Magnetics Conf. Dig. (IEEE Intermag 2000), Apr. 9-13, 2000, pp. AS-09-AS-09.

[23] M. Sendoh, K. Ishiyama, and K. I. Arai, "Direction and individual control of magnetic micromachine," in Proc. 2002 Int. Magnetics Conf. (IEEE Intermag 2002), Apr--May 2002, pp. 3356-3358.

[24] M. Sendoh, K. Ishiyama, K. I. Arai, M. Jojo, F. Sato, and H. Matsuki, "Fabrication of magnetic micro-machine for local hyperthermia," in Proc. 2002 IEEE International Magnetics Conf. (Intermag 2002), Apr--May 2002, pp. FU11-FU11.

[25] K. B. Yesin, K. Vollmers, and B. J. Nelson, "Analysis and design of wireless magnetically guided microrobots in body fluids," in Proc. 2004 IEEE Int. Conf. Robotics and Automation, Apr-May 26-1, 2004, pp. $1333-1338$.

[26] F. M. White, Viscous Fluid Flow, ser. Mechanical Engineering. New York: McGraw Hill, 1991.

[27] P. Kundu and I. Cohen, Fluid Mechanics, 2nd ed: Academic, 2002.

[28] V. Fidleris and R. L. Whitmore, "Experimental determination of the wall effect for spheres falling axially in cylindrical vessels," Br. J. Appl. Phys., vol. 12, pp. 490-494, Sep. 1961.

[29] P. H. T. Uhlherr and R. P. Chhabra, "Wall effect for the fall of spheres in cylindrical tubes at high Reynolds number," Can. J. Chem. Eng., vol. 73, no. 6, pp. 918-923, 1995.

[30] R. Kehlenbeck and R. Di Felice, "Empirical relationships for the terminal settling velocity of sheres in cylindrical columns," Chem. Eng. Technol., vol. 21, pp. 303-308, 1999.

[31] R. M. Wham, O. A. Basaran, and C. H. Byers, "Wall effects on flow past fluid spheres at finite Reynolds number: Wake structure and drag correlations," Chem. Eng. Sci., vol. 52, no. 19, pp. 3345-3367, Oct. 1997.

[32] Munroe, "The english versus the continental system of jigging-Is close sizing advantageous?," Trans. Am. Inst. Mining Eng., vol. 17, pp. 637-637, 1888.

[33] J.-B. Mathieu, S. Martel, L. Yahia, G. Soulez, and G. Beaudoin, "Preliminary investigation of the feasibility of magnetic propulsion for future microdevices in blood vessels," Biomed. Mater. Eng., to be published.

[34] (2004) Princeton Applied Research. Princeton Applied Research Home. [Online]. Available: http://www.princetonappliedresearch.com

[35] (2004) Sypris Test \& Measurement. Sypris Test \& Measurement/F.W. Bell Products. [Online]. Available: http://www.fwbell.com/

[36] (2004) March Pumps. March Magnetic Pumps. [Online]. Available: http://www.marchpump.com/

[37] (2004) Stainless-Steel $65 \mathrm{~mm}$ \& 150-mm Calibrated/Direct Reading. Barnant Company. [Online]. Available: http://www.barnant.com/gilmont/prod707.htm

[38] F. M. White, Fluid Mechanics, 4th ed. New York: McGraw Hill, 1999.

[39] (2004) Philips Medical. Philips, Magnetic Resonance Imaging-Achieva 1.5 T. [Online]. Available: http://www.medical.philips.com/main/products/mri/products/achieva15T/index.html

[40] W. R. Milnor, Hemodynamics. Baltimore, MD: Williams and Wilkins, 1982.

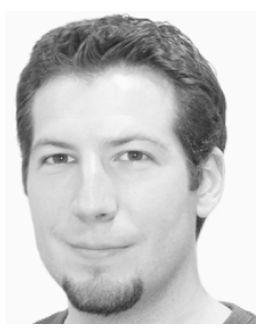

Jean-Baptiste Mathieu received the B.Sc. degree in mechanical engineering in 2002 and the M.S. degree in biomedical engineering from Ecole Polytechnique de Montréal (EPM), Montréal, QC, Canada, in 2004.

$\mathrm{He}$ is currently with the NanoRobotics Laboratory in the department of Computer Engineering at EPM. His current research interests are in the development of medical applications of microdevices propelled in the blood vessels by magnetic gradients generated by magnetic resonance imaging (MRI) systems. 


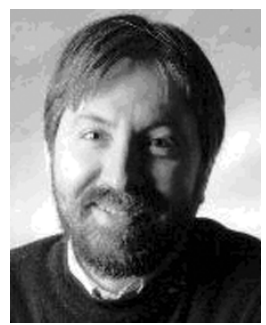

Gilles Beaudoin (S'90-M'90) received the Ph.D. degree in experimental high energy physics from the University of Montréal, Montréal, QC, Canada, in 1990.

For the first few years, he worked in detector development with the OPAL and ATLAS collaborations at CERN. Since 1996, he is at the Centre Hospitalier de l'Université de Montréal, Montréal, where he works in the imaging aspect of medical physics. He is head of the masters degree program in medical physics at the University of Montréal, a new program that he helped instigate. Since 2002, he is President of the Association Québécoise des Physicien(ne)s Médicaux Cliniques, Québec, Canada. His research interests are functional and interventional MRI and X-ray detection systems.

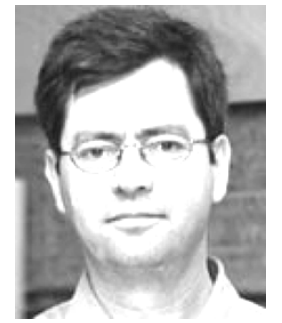

Sylvain Martel (S'95-M'96) received the B.E. degree from the Université du Québec (UQTR), Québec, and the M.Eng. and Ph.D. degrees from McGill University, Montréal, QC, Canada, in 1989 and 1997, respectively, all in electrical engineering.

Following postdoctoral studies at The Massachusetts Institute of Technology (MIT), he was Research Scientist at the BioInstrumentation Laboratory, Department of Mechanical Engineering at MIT He is currently Associate Professor in the Department of Computer Engineering and the Institute of Biomedical Engineering at École Polytechnique de Montréal (EPM), Montréal, and Director of the NanoRobotics Laboratory at EPM. He holds the Canada Research Chair (CRC) in Micro/Nanosystems, Development, Construction, and Validation. He has over 90 refereed publications and participates in many international committees and organizations Presently, Dr. Martel leads a multidisciplinary team involved in research and development of new platforms and nano-factories based on a fleet of scientific instruments configured as autonomous miniature robots capable of high throughput autonomous operations at the molecular scale, minimally invasive tools based on microdevices propelled in the blood vessels by magnetic gradients generated by magnetic resonance imaging (MRI) systems, microelectromechanical system (MEMS), and System-on-Chip (SoC)based miniature instrumented robots, development of new MEMS/NEMS based on the integration of bacteria as biological components, and many other related projects. Dr. Martel's main expertise is in the field of nanorobotics, micro- and nano-systems, and the development of novel instrumented platforms including advanced micromechatronics systems and a variety of related support technologies. He has also a vast experience in electronics, computer engineering, and also worked extensively in biomedical and mechanical engineering. 